\title{
Inhibition of SIRT2 in merlin/NF2-mutant Schwann cells triggers necrosis
}

\author{
Alejandra M. Petrilli ${ }^{1}$, Marga Bott ${ }^{1}$, and Cristina Fernández-Valle ${ }^{1}$ \\ ${ }^{1}$ Department of Biomedical Science, College of Medicine, University of Central Florida, Lake Nona-Orlando, Florida, USA \\ Correspondence to: Cristina Fernández-Valle, email: cfv@ucf.edu \\ Keywords: Neurofibromatosis Type2; high-throughput screen; SIRT2; acetylation, merlin, tumor suppressor, AGK2 \\ Received: September 18, 2013 Accepted: November 14, $2013 \quad$ Published: November 15, 2013
}

This is an open-access article distributed under the terms of the Creative Commons Attribution License, which permits unrestricted use, distribution, and reproduction in any medium, provided the original author and source are credited.

\section{ABSTRACT:}

Mutations in the NF2 gene cause Neurofibromatosis Type 2 (NF2), a disorder characterized by the development of schwannomas, meningiomas and ependymomas in the nervous system. Merlin, a tumor suppressor encoded by the NF2 gene, modulates activity of many essential signaling pathways. Yet despite increasing knowledge of merlin function, there are no NF2 drug therapies. In a pilot high-throughput screen of the Library of Pharmacologically Active Compounds, we assayed for compounds capable of reducing viability of mouse Schwann cells (MSC) with Nf2 inactivation as a cellular model for human NF2 schwannomas. AGK2, a SIRT2 (sirtuin 2) inhibitor, was identified as a candidate compound. SIRT2 is one of seven mammalian sirtuins that are NAD+-dependent protein deacetylases. We show that merlin-mutant MSC have higher expression levels of SIRT2 and lower levels of overall lysine acetylation than wild-type control MSC. Pharmacological inhibition of SIRT2 decreases merlin-mutant MSC viability in a dose dependent manner without substantially reducing wild-type MSC viability. Inhibition of SIRT2 activity in merlin-mutant MSC is accompanied by release of lactate dehydrogenase and high mobility group box 1 protein into the medium in the absence of significant apoptosis, autophagy, or cell cycle arrest. These findings suggest that SIRT2 inhibition triggers necrosis of merlin-mutant MSCs and that SIRT2 is a potential NF2 drug target.

\section{INTRODUCTION}

Neurofibromatosis type 2 (NF2) is a benign tumor disorder of the nervous system caused by mutations in the NF2 gene that encodes a tumor suppressor called schwannomin or merlin. The hallmark of NF2 is the formation of bilateral schwannomas in the vestibular branch of the auditory nerve. Patients frequently develop additional schwannomas in other cranial, spinal and peripheral nerves as well as meningiomas and ependymomas. Common initial symptoms include hearing loss, dizziness or imbalance; however life-threatening compression of the brainstem also occurs [1]. The choices for NF2 schwannoma treatments are surgical resection or stereotactic radiosurgery. Many schwannomas however are inoperable and surgery often causes complete loss of nerve function, while radiosurgery carries an increased risk of a future secondary malignancy [2]. Currently, a few clinical trials of anti-cancer drugs are underway for NF2 [3, 4].
Molecular studies of merlin's mechanism of action have revealed that merlin regulates signaling from mitogenic, adhesion and extracellular matrix receptors through many essential signaling pathways $[5,6]$. However, the pleotropic effect of merlin has made it difficult to identify the most relevant drug targets.

As an alternative approach to drug discovery, we conducted an unbiased high-throughput screen of the library of Pharmacologically Active Compounds (LOPAC) using viability of merlin-mutant mouse Schwann cells (MSC) as a phenotypic assay to identify potential compounds and pathways relevant to NF2 schwannoma treatment. One compound identified in the screen was AGK2, a SIRT2 inhibitor. SIRT2 is one of seven mammalian sirtuins, also known as class III HDACs (histone deacetylases). Sirtuins are $\mathrm{NAD}(+)$ dependent deacetylases, that remove the acetyl group from the lysine's epsilon-amine in a multi-step reaction[7, 8]. SIRT2 is mainly cytoplasmic and its known substrates 
include: $\alpha$-tubulin, partitioning defective 3 homolog (PAR3), p53, K-RAS, histone H4K16, forkhead Box O1 and $3 \mathrm{a}$ (FOXO1 and 3a) and RIP1 [9-14]. While beneficial effects of SIRT2 inhibition was shown in neurodegenerative diseases such as Parkinson's and Huntington's disease, the role of SIRT2 in cancer remains controversial $[15,16]$. SIRT2 has been reported to function as a tumor suppressor that is down-regulated in some human gliomas; however, its function has also been reported as essential for survival of C6 glioma cells. Small molecule SIRT2 inhibitors have in some cases selectively induced tumor cell death [17-21].

Here we validate AGK2 as a compound that selectively reduces viability of merlin-mutant MSC compared to normal MSCs. Moreover we demonstrate increased expression levels of SIRT2 in merlin-mutant versus normal MSCs that are associated with a general reduction in lysine acetylation. Phenotypic mechanism of action studies suggests that inhibition of SIRT2 in merlinmutant SCs triggers a necrotic pathway.

\section{RESULTS}

\section{Merlin-Mutant MSC Have Higher SIRT2 Levels and Lower Lysine Acetylation Levels Than Control MSC.}

Merlin-mutant mouse Schwann cells (MSC) contain a deletion of exon 2 of the $N f 2$ gene that replicates a documented patient mutation. Merlin-mutant MSC were created by in vitro adeno-Cre transduction of mouse Schwann cells isolated from sciatic nerves of homozygous $N f 2^{f l o x z / f o x z}$ mice as previously described [2224]. Using this NF2 cell model, we screened the Library of Pharmacologically Active Compounds (LOPAC, Sigma-Aldrich) searching for compounds capable of reducing their viability. One of the initial hits we chose to investigate was AGK2, a small molecule SIRT2 inhibitor. The sirtuin family of deacetylases has not been examined heretofore in NF2.

To visualize the lysine acetylation patterns in merlinmutant MSC and control MSC, we performed a western blot using an $\varepsilon$-acetyl-Lysine antibody. Control MSC had a higher number of and more intensely acetylated bands than merlin-mutant MSC (Fig. 1a and Supplementary Fig. S1). Because the merlin-mutant MSC presented fewer bands, we asked whether SIRT2, the target of AGK2 had altered expression levels. Indeed we found that merlinmutant MSC expressed SIRT2 at higher levels than control MSC (Fig. 1b). The acetyl-lysine blot differed greatly around the $50 \mathrm{kDa}$ molecular weight marker. Because SIRT2 is a recognized acetyl- $\alpha$-tubulin deacetylase, we investigated if any of those bands were $\alpha$-tubulin. Merlinmutant MSC have highly deacetylated tubulin levels compared to control MSC (Fig. 1b). We confirmed these differences with several independently derived merlinmutant MSC lines (Fig. 1c). We next immunoblotted for glyceraldehyde-3-phosphate dehydrogenase (GAPDH) because tumor cells have altered metabolism and sirtuins can influence metabolic and energetic regulation. We found higher levels of GAPDH in merlin-mutant MSC compared to MSC consistent with higher glycolytic activity in merlin-mutant MSC than controls (Fig. 1c). In order to validate the results in our cellular model, we measured SIRT2 and acetyl- $\alpha$-tubulin levels in a human NF2 cell line created by immortalization of schwannoma cells from a NF2 patient with the E6 and E7 genes of the papillomavirus [25]. Similarly, we found higher levels of SIRT2 in HEI-193 cells than in control human Schwann cells which correlated with lower acetylated tubulin levels in HEI-193 compared to control cells (Fig. 1d).

We localized SIRT2 in control and mutant cells using immunofluorescence staining followed by confocal microscopy. Both cells types expressed the Schwann cell marker S100 and control MSC immunoreacted with the $\mathrm{N}$-terminal merlin antibody whereas the merlin-mutant MSC did not. Both cell types expressed SIRT2 in the cytosol and nuclei, however, merlin-mutant MSC in general had higher levels of fluorescence intensity than controls. Control MSC showed significantly higher levels of acetylated tubulin compared to merlin-mutant MSC and in the latter, the weak immunofluorescence signal for acetylated tubulin was mostly perinuclear (Fig. 1e).

Lastly, we examined the expression of other sirtuin family members by western blotting. SIRT5 was expressed in control and mutant MSC at similar levels, whereas SIRT1, SIRT3 and SIRT7 were expressed at higher levels in merlin-mutant MSC than MSC (Fig. 1f).

\section{SIRT2 Inhibition Selectively Reduces Merlin- Mutant MSC Viability in a Dose-Dependent Manner}

To evaluate selectivity of SIRT2 inhibition for merlin-mutant MSC viability, we conducted a dose response study of AGK2 using CellTiter-Fluor assay. We found that a 24 hour exposure to AGK2 decreased merlin-mutant MSC viability in a dose-dependent manner with an $\mathrm{IC}_{50}=9.0 \mu \mathrm{M}$ (Fig. 2a). In contrast, AGK2 did not decrease control MSC viability as effectively as for merlin-mutant cells (Fig. 2b). At $10 \mu \mathrm{M}$ AGK2, merlinmutant cells retained $45.8 \pm 0.7 \%$ viability compared to control MSC that retained $70.9 \pm 1.8 \%$ viability.

Although AGK2 is a highly selective SIRT2 inhibitor, it does minimally inhibit SIRT1 and SIRT3 at ten times the SIRT2 $\mathrm{IC}_{50}$ level when tested in vitro [15]. To corroborate that SIRT2 was specifically inhibited by AGK2 and associated with loss of merlin-mutant MSC viability, we tested the effect of an alternative SIRT2 
inhibitor, AK1. This benzylsulfonamide also inhibits SIRT2 enzymatic activity by targeting the nicotinamide binding site but with less potency for SIRT1 and SIRT3 than AGK2. We found that AK1 decreased merlin-mutant MSC viability in a dose-dependent manner with an $\mathrm{IC}_{50}=$

a

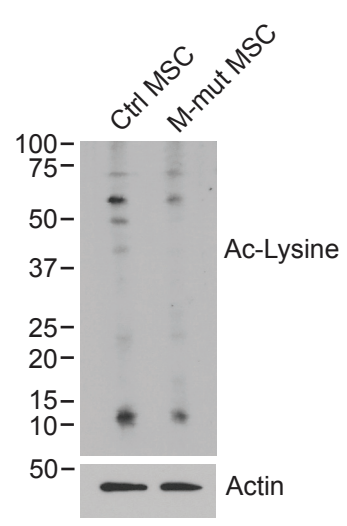

b

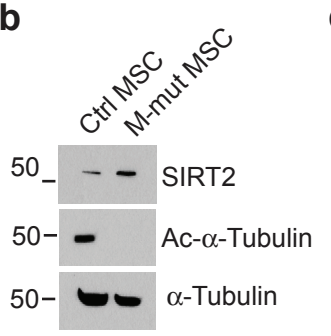

d

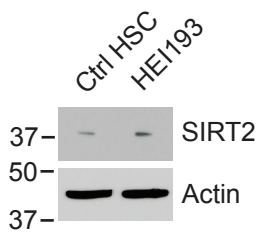

$26.1 \mu \mathrm{M}$ (Fig. 2c). AK1 minimally reduced control MSC viability which retained $82.8 \pm 2.1 \%$ viability at $25 \mu \mathrm{M}$ (Fig. 2d). These results suggest that pharmacological inhibition of SIRT2 selectively reduced merlin-mutant MSC viability.
C

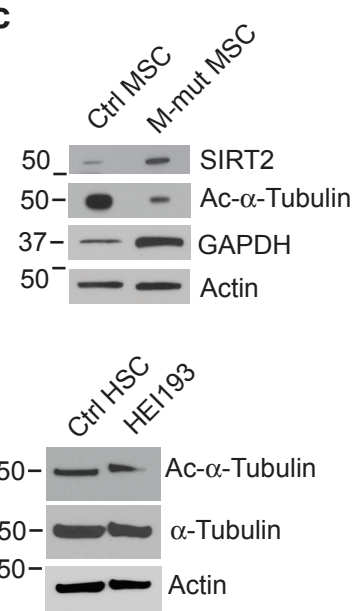

e

Ctrl MSC

M-mut MSC
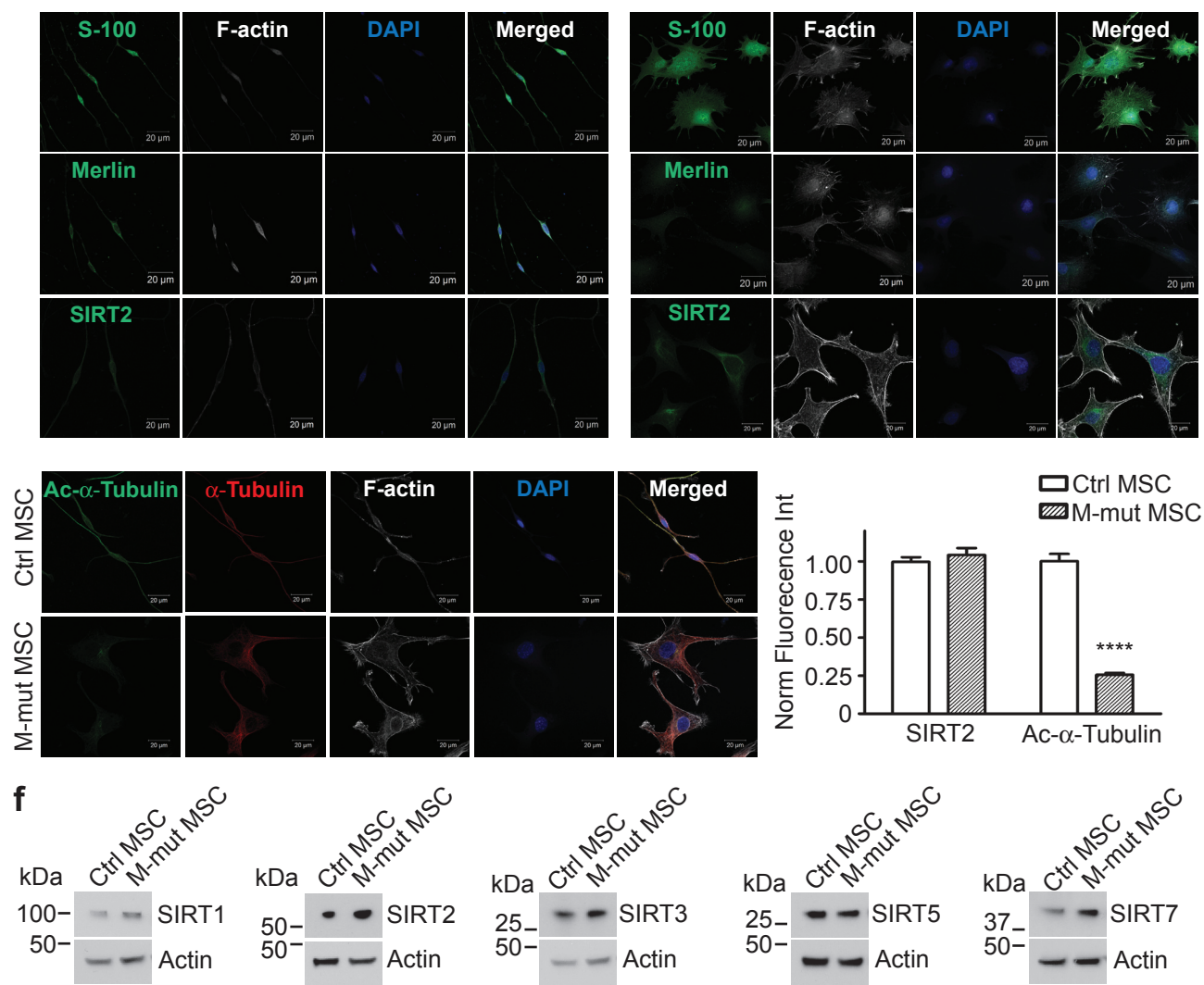

Figure 1: Merlin-Mutant MSC (M-mut MSC) Have Lower Levels of Lysine Acetylation and Higher Levels of SIRT2 Compared to Control MSC. Control $N f 2^{f f o x z / f o x 2}$ MSC and merlin-mutant MSC were analyzed by western blotting for: a) Acetyllysine, b) SIRT2, acetyl- $\alpha$-tubulin, $\alpha$-tubulin and c) SIRT2, GAPDH. Anti- $\beta$-actin was used as a loading control. d) Cultured control human Schwann cells (HSCs) from normal individuals and HEI193 cells were analyzed by western blotting for SIRT2, acetyl- $\alpha$-tubulin, $\alpha$-tubulin. Anti- $\beta$-actin was used as a loading control. e) Representative confocal images of control MSC and merlin-mutant MSC grown overnight on glass coverslips and immunostained with the indicated antibodies (green). The nucleus was visualized with DAPI stain (blue) and F-actin with phalloidin-Alexa633 (white). Scale bar: $20 \mu \mathrm{m}$. Quantitation of the immunofluorescence from three independent experiments was performed with Volocity software. ${ }^{* * * *} P<0.0001$ determined by two-way ANOVA using Bonferroni post-tests. f) Control $N f 2^{f f o x} 2$ fox 2 MSC and merlin-mutant MSC were analyzed by western blotting for SIRT1, SIRT2, SIRT3, SIRT5, SIRT7 and $\beta$-actin. 


\section{SIRT2 Inhibition Reduces Merlin-Mutant MSC Proliferation Without Interfering with Cell Cycle Progression or DNA Synthesis}

We next assessed the ability of AGK2 to decrease cell viability for longer incubation times. We treated merlin-mutant MSC with vehicle or inhibitor and assessed the cell number by crystal violet staining at 24,48 and 72 hours. We found that at 72 hours AGK2 significantly reduced the number of merlin-mutant MSC compared to vehicle control (Fig. 3a). We also measured EdU incorporation in AGK2-treated merlin-mutant MSC and found that it did not decrease DNA synthesis compared to vehicle treated controls (Fig. 3b).

To determine if the decrease in cell number caused by AGK2 was associated with inhibition of cell cycle progression, we performed flow cytometry analysis of a

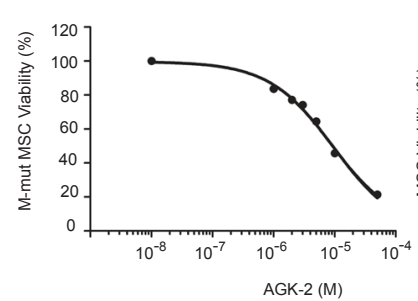

c

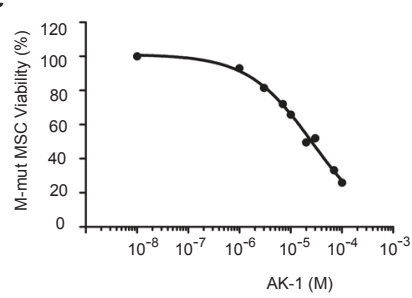

b
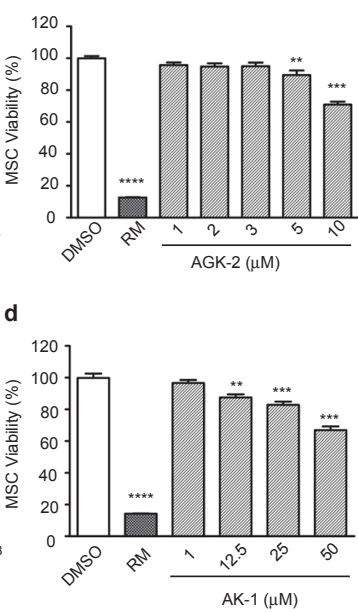

Figure 2: SIRT2 Inhibition With AGK2 and AK1 Selectively Decreases Merlin-Mutant MSC Viability. a) AGK2 dose response curve for cell viability. Merlin-mutant MSC were seeded at 5,000 cells/well in 384-well dishes and were incubated with increasing concentrations of AGK2 for 24 hours. Cell viability was assessed with the CellTiter-Fluor assay. DMSO control was considered $100 \%$ viability. Rapamycin (RM) $(50 \mu \mathrm{M})$ was used as positive control for cell death. Graph represents the mean \pm SEM of 3 independent experiments analyzed together $(\mathrm{n}=96), \mathrm{IC}_{50}=9.01 \mu \mathrm{M}, \log$ [inhibitor] vs. response, variable slope (four parameters). b) AGK2 dose response for control MSC viability. Cell viability was measured as in (a). Graph represents the mean $\pm \operatorname{SEM}(n=36)$. $* * P<0.01$; $* * * P<0.001 ; * * * * P<0.0001$ determined by one-way ANOVA using Turkey's multiple comparison test. c) AK1 dose response curve for merlin-mutant MSC viability. Cell viability assessed as in (a). Graph represents the mean \pm SEM of 3 independent experiments analyzed together $(n=96), \quad \mathrm{IC}_{50}=26.1 \mu \mathrm{M}, \log$ [inhibitor] vs. response, variable slope (four parameters). d) AK1 dose viability response for control MSC. Cell viability was measured as in (a). Graph represents the mean \pm SEM $(n=20)$. $* * * P<0.001 ; * * * * P<0.0001$ determined by one-way ANOVA using Turkey's multiple comparison test. propidium iodine (PI) labeled cells. We found that AGK2 did not significantly alter the distribution of diploid cells in the cell cycle, although there was a tendency to slightly increase the percentage of cells in the G2/M phase (Fig. $3 e)$. To further analyze the effect of AGK2 on cell cycle progression, we performed a BrdU/7-AAD assay. There was no significant change in the distribution of treated and untreated cell populations during the cell cycle after 24 hours (Fig. 3f). These results suggest that SIRT2 inhibition decreased merlin-mutant MSC proliferation by mechanisms independent of interfering with cell cycle progression.

\section{SIRT2 Inhibition Induces Merlin-Mutant MSC Cell Death}

To determine if SIRT2 inhibition reduced merlinmutant MSC viability by inducing apoptosis, we tested caspase 3/7 activity following AGK2 treatment. We found that AGK2 moderately increased caspase $3 / 7$ activity at 10 $\mu \mathrm{M}$ compared to the positive control, staurosporine, that increased caspase 3 and 7 activity to much higher levels in a dose-dependent manner (Fig. 4a). To further analyze if SIRT2 inhibition induced caspase independent apoptosis, we studied the effect of AGK2 on merlin-mutant MSC membrane asymmetry using the violet ratiometric flow cytometry assay. We found AGK2 only moderately increased apoptosis from $3.5 \pm 1.2 \%$ in merlin-mutant MSC treated with DMSO to $6.1 \pm 2.8 \%$ when treated with AGK2. This increase however was not statistically significant (Fig. 4b,c). These results suggest that even though AGK2 slightly induced apoptosis it is not the main mechanism responsible for the decreased viability of merlin-mutant MSC.

Next, we assessed whether SIRT2 inhibition decreased merlin-mutant MSC viability by inducing cell death through necrosis. We measured cytotoxicity with a fluorescence plate format assay that uses a cyanine dye impermeant to live cells that stains DNA from dead cells and increases its fluorescence. We found that both, AGK2 and AK1, significantly increased the number of dead merlin-null MSC in dose-dependent manners (Fig. $5 a, b)$. To further analyze the mechanism of cell death, we measured the release of lactate dehydrogenase (LDH) from cells with damaged membranes into the medium with the CytoTox-ONE homogeneous membrane integrity assay. We found that both SIRT2 inhibitors, AGK2 and AK1 increased the levels of LDH released to the medium in a dose dependent manner. This is typically associated with cell necrosis (Fig. 5c,d). We also measured release from cells of the necrosis marker high mobility group box 1 protein (HMGB1) by western blot [26]. We found that AGK2 and AK1 induced release of significant amounts of HMGB1 into the medium that corresponded with a decrease in intracellular HMGB1 levels (Fig. 5e). To 
further characterize merlin-mutant MSC cell death, we assessed the induction of autophagy by immunoblotting for lipidated microtubule-associated protein 1 Light Chain 3 B (LC3B-II). Cytosolic LC3B-I during autophagy is lipidated by Atg 7 and 3 and is converted in LC3B-II that associates with autophagic vesicles [27]. We found that although DMSO slightly activates autophagy, neither AGK2 nor AK1 induced it (Fig. 5f).

These results suggest that SIRT2 inhibition decreased merlin-null MSC viability by triggering cell death characterized by release of LDH and HMGB1.

\section{DISCUSSION}

\section{SIRT2 and Schwann Cells}

SIRT2 was identified as a potential NF2 drug target from a pilot LOPAC screen. We confirmed that SIRT2 a

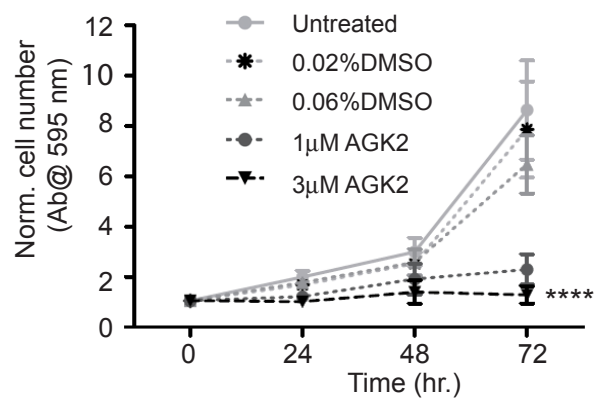

。

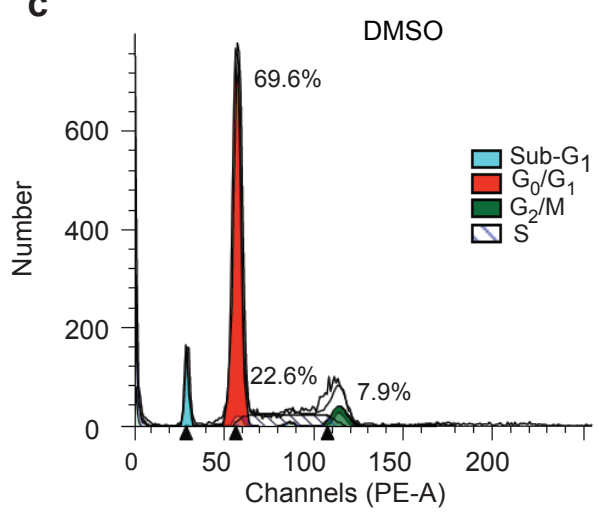

b

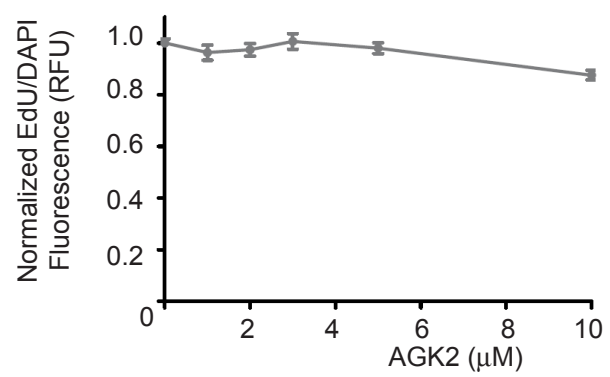

AGK2

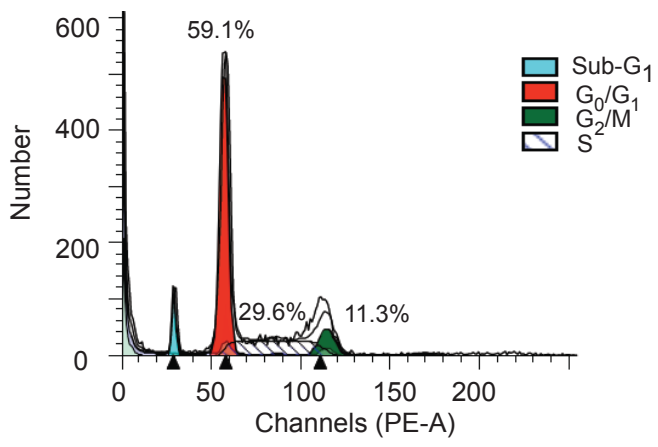

d
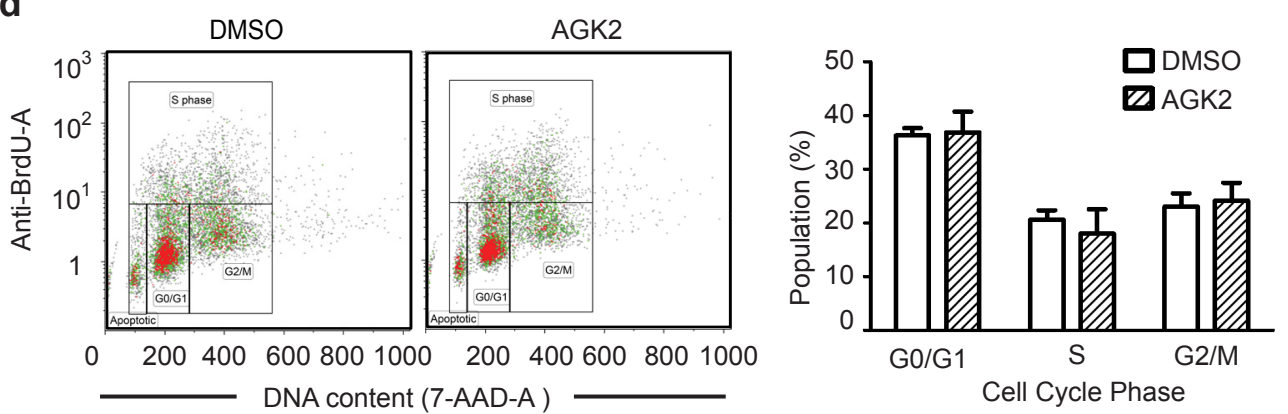

Figure 3: AGK2 Decreases Merlin-Mutant MSC Proliferation Without Interfering With Cell Cycle Progression. a) Merlin-mutant MSC were incubated with AGK2 or DMSO for the indicated times and the number of cells was assessed using a crystal violet assay. Graph combines 3 independent experiments in triplicates (mean \pm SEM, $n=9$ ). b) Merlin-mutant MSC were treated with increasing AGK2 concentrations for 24 hours. For the last 6 hours $10 \mu \mathrm{M}$ EdU was added to the culture. Incorporation of EdU into S-phase cells was assessed with the EdU Click-It microplate assay. Graph combines 3 independent experiments (mean $\pm \mathrm{SEM}$, $\mathrm{n}=44$ ). c) Merlinmutant MSC were treated with $5 \mu \mathrm{M}$ AGK 2 for 8 hours prior to propidium iodide labeling. Cells were analyzed by flow cytometry and the diploid cell population was evaluated with the ModFit program. d) Merlin- mutant MSC were treated with AGK2 or DMSO control overnight and with $10 \mu \mathrm{M}$ BrdU for the last $3 \mathrm{hr}$ of incubation. Cells were analyzed by flow cytometry. Plot of the distribution of BrdU- and 7-AAD-labelled cells analyzed with Kaluza software. These distribution plots are representative of 3 independent experiments ( $\mathrm{n}=3$ ). Bar graph of the distribution of the cell cycle phases of the experiments as mean \pm SEM. 
protein levels were higher in cultured merlin-mutant MSC compared to cultured control MSC and that SIRT2 activity was essential for survival of merlin-mutant Schwann cells but not wild type MSC. SIRT2 inhibition by AGK2 and AK1 selectively decreased viability of Schwann cells lacking functional merlin. The AGK2 and AK1 dose-dependent loss of cell viability correlated with a dose-dependent cell death that was further characterized as associated with release of the necrotic markers LDH and HMGB1 without induction of autophagy, cell cycle arrest or significant caspase 3/7 dependent or independent apoptosis.

Although SIRT2 has been considered a potential regulator of cell cycle progression [28-30], we did not

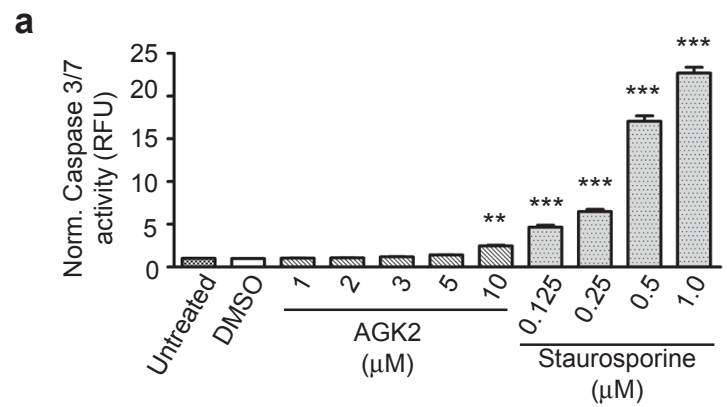

b

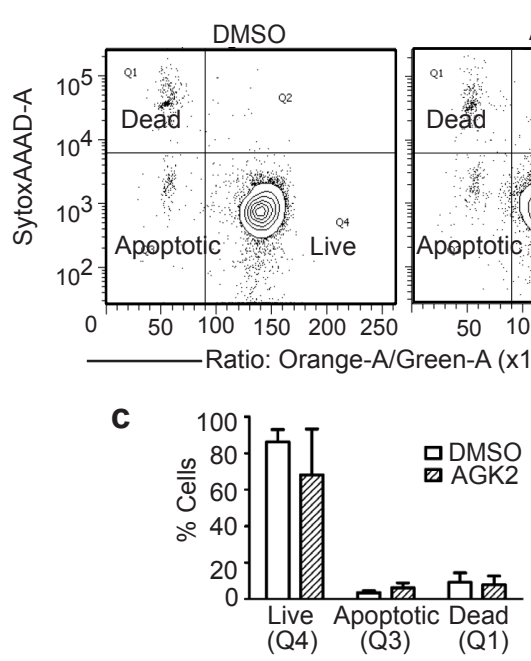

Figure 4: SIRT2 Iinhibition With AGK2 Does Not Induce Significant Apoptosis. a) Caspase 3/7 activity assay. Merlin-mutant MSC were seeded in 384 well plates at 5,000 cells/well in $20 \mu \mathrm{l}$ phenol-red free medium. After 16 hours 5 $\mu 1 /$ well of inhibitor/vehicle was added and incubated for an additional 8 hours. Activity of the Caspase 3/7 was measured with the ApoONE Homogeneous assay. Staurosporine curve was used as positive control. Histogram represents 3 independent experiments $(\mathrm{n}=96)$ normalized to untreated and analyzed together. $\quad * * P<0.05 ; \quad * * * P<0.001$ determined by one-way ANOVA using Dunnett's multiple comparison test to DMSO control. b) Plasma membrane asymmetry was evaluated with the Violet ratiometric assay by flow cytometry. Merlin-mutant MSC were incubated with AGK2 or DMSO vehicle for 24 hours and analyzed by flow cytometry. Densitometry graph: apoptotic (Q3), dead (Q1), live (Q4). Below, bar graph represents mean \pm SEM of 3 independent experiments $(n=3)$. find evidence for that mechanism of action in this study. SIRT2 was reported to associate with mitotic structures and to increase in abundance and phosphorylation during mitosis. It has been reported to prevent chromosome condensation and entry into $M$ phase in response to mitotic stress. Additionally, overexpression of catalytically inactive SIRT2 increased the number of multinucleated HeLa cells. However in the absence of stressors, SIRT2 activity was not required for cell cycle progression in HeLa and HEK293 cells [31]. In Schwann cells, selective inactivation of Sirt2 during early embryogenesis did not reduce the number of Schwann cells produced during the period of rapid cell proliferation that occurs at post-natal days 1-5. This supports the conclusion that cell cycle progression in Schwann cells is independent of SIRT2 activity [9] in agreement with our finding that SIRT2 inhibition did not decrease merlin-mutant MSC viability by arresting cell cycle progression but rather by inducing cell necrosis. There is evidence for a role of SIRT2 in necrosis. Nie et al. reported that AGK2 treatment of PC12 cells decreased intracellular ATP levels and increased necrosis without affecting autophagy [32]. Additionally, tumor necrosis factor alpha (TNF- $\alpha$ ) was shown to activate necroptosis via deacetylation of receptor-interacting protein 1 (RIP1) by receptor-interacting protein 3 (RIP3) bound SIRT2 allowing the formation of a stable complex in L929 and Jurkat T cells [14]. Due to the prominent role of cytokines in $\mathrm{SC}$ development and repair others have shown that while a low concentration of TNF- $\alpha$ induced SC proliferation, a high concentration of TNF- $\alpha$ induced SC growth arrest and apoptosis [33-35]. We have not studied TNF- $\alpha$ in merlin-mutant Schwann cells and additional studies are needed to identify the pathway by which inhibition of SIRT2 activity leads to cell necrosis.

\section{SIRT2 in Cancer}

Similar to our findings in Schwannoma and control Schwann cells, upregulation of SIRT2 mRNA and protein levels has been reported in some cancer cells such as primary acute myeloid leukemia blasts compared to control hematopoietic progenitor cells from healthy individuals [36]. In glioma cells, however, it has been reported that several human glioma samples had reduced SIRT2 mRNA compared to normal brain tissue and that overexpression of SIRT2 decreased HTB14 glioma cells colony formation [18]. Notably, inhibition of SIRT2 activity decreased C6 glioma cells viability [17]. Additionally, aged mice with inactivation of the Sirt2 gene by deletion of exons 5-8 developed different types of tumors depending on their gender [19]. Treatment of C6 glioma cells with $10 \mu \mathrm{M}$ AGK2 for 24 hours caused a $60 \%$ loss of viability due to both apoptosis and necrosis [17]. Similarly, another SIRT2 inhibitor, compound AC93253 showed selectively decreasing viability in several human cancer cell lines over their human primary cells 
controls [21]. The panel included cell lines from prostate, pancreas, cervical and lung cancer. AC-93253 was also selectively cytotoxic to HeLa cells by inducing apoptosis and necrosis. Discrepancies in the positive and negative associations of SIRT2 with tumor development are likely due to differences in cell type, developmental activation patterns for SIRT2 and substrate preferences [9, 37]. For instance histone $\mathrm{H} 4$ is a principal substrate for SIRT2 in various cell types but not in oligodendrocytes $[11,38]$.
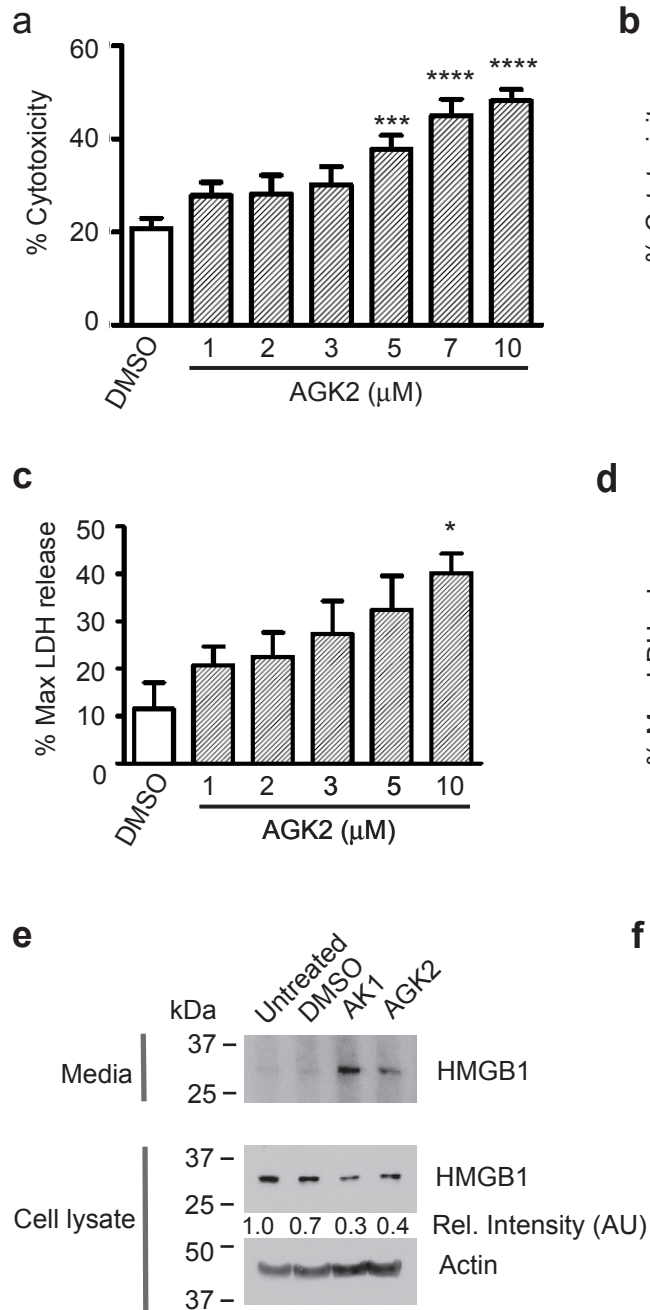

b

d

f
Although AGK2 and AK1 selectively inhibit SIRT2, at higher concentrations they can also inhibit SIRT1 and SIRT3 in vitro [15]. Therefore it is possible that at the $\mathrm{IC}_{50}$ concentrations used in this study, the compounds could have also slightly decreased SIRT1 and 3 activity. Interestingly, treatment of BCL6expressing Burkitt lymphoma cells and Burkitt lymphoma xenograft mice with a dual SIRT1 and SIRT2
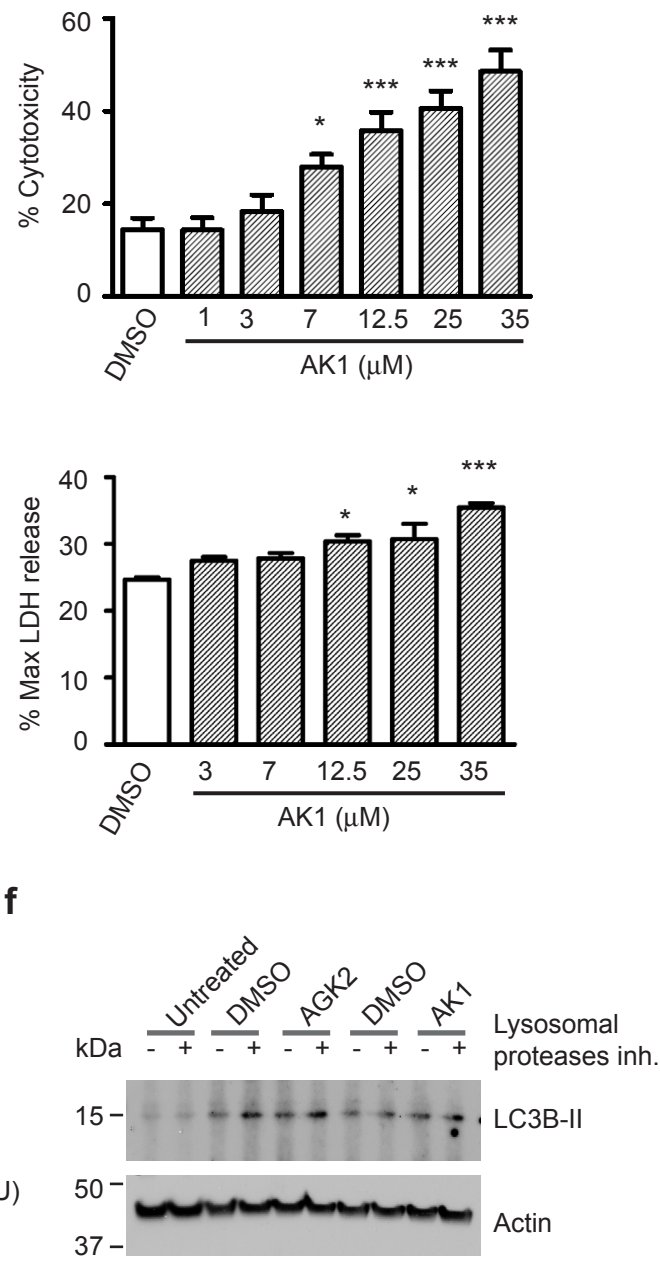

Figure 5: SIRT2 Inhibition Causes Release of LDH and HMGB1 from Merlin-Mutant MSC. Merlin-mutant MSC were seeded in 384 well-plates at 5,000 cell/well with cellTox-Green dye in phenol-red free medium. After attachment cells were treated with increasing concentrations of a) AGK2 and b) AK1 and incubated for 24 hours. Cytotoxicity was measure in a plate reader. Digitonin treatment was considered $100 \%$ cytotoxicity control. $* P<0.05$; $* * * P<0.001 ; * * * * P<0.0001$ determined by one-way ANOVA using Bonferroni's multiple comparison test. c) Merlin-mutant MSC (5,000 cells/well in a 384-well plate) were treated for 4 hours with increasing concentrations of AGK2 and release of LDH into the medium was measured with a fluorogenic assay, the CytoTox-ONE homogenous membrane integrity assay in a plate reader; digitonin was considered as control for maximum LDH release. ${ }^{*} P<0.05$ determined by oneway ANOVA using Dunnett's multiple comparison test d) Assessment of LDH released to the medium by merlin-mutant MSC treated 4 hours with increasing concentrations of AK1 as in (c), ${ }^{*} P<0.05 ; * * * P<0.001$ determined by one-way ANOVA using Dunnett's multiple comparison test. e) Merlin-mutant MSC were seeded in 6-well plates and treated for 6 hours with inhibitors/vehicle. HMGB1 release into the medium and cellular content of HMGB1 was assessed by western blot with anti-HMBG1 antibody. Anti- $\beta$-actin was used as loading control. f) Induction of autophagy was assessed by LC3B-II immunobloting. Merlin-mutant MSC were treated for 3 hours with AGK2 or AK1 plus and minus $10 \mu \mathrm{g} / \mathrm{ml}$ acidic lysosomal proteases inhibitors (E64-d and pepstatin-A). Cell were lysed in 1\% SDS buffer and resolved by western blot for anti-LC3B. Anti- $\beta$-actin was used as loading control. 
inhibitor, cambinol, produced a potent antitumor effect [39]. Hence, there may be value to SIRT2 and SIRT1 combinatorial inhibition in schwannoma treatment. A recent study showed that acetylation of K-RAS at lysine104 attenuates its transforming activity in cancer cells and both SIRT2 and HDAC6 deacetylate K-RAS [12]. Moreover, $\alpha$-tubulin acetylation and cortactin are regulated by SIRT2 and HDAC6 [10, 40, 41]. Due to the increasing interest of HDAC inhibitors in cancer, a new pan-HDAC inhibitor AR42 was tested on merlin mutant schwannoma and meningioma cells. Both in vitro and in vivo treatment showed a selective anti-proliferative effect on schwannoma and meningioma cells warranting further clinical evaluation for NF2 related tumors [42-44]. AR42, similar to other HDAC inhibitors also decreased Akt phosphorylation, a pathway upregulated in many cancers and NF2. Chen et al. have shown that AR42 targets HDAC1 and HDAC6 by disrupting their HDAC-protein phosphatase 1 (PP1) complexes, which leads to increased PP1-Akt association, and facilitates PP1-mediated dephosphorylation of Akt [45]. Therefore combinatorial inhibition of SIRT2 and HDAC6 could be evaluated when considering modulation of acetylation signaling for NF2 treatment.

We speculate that SIRT2 pharmacological inhibition may have some therapeutic value for NF2-associated schwannomas by promoting necrosis. However, additional research is needed to understand the relationship between merlin and SIRT2 in normal Schwann cells and the effect of merlin inactivation on protein acetylation and cell survival.

\section{MATERIALS AND METHODS}

\section{Inhibitors}

The SIRT2 inhibitors AGK-2; CAS name 2-Cyano3-[5-(2,5-dichlorophenyl)-2-furanyl]-N-5-quinolinyl2-propenamide and AK1; CAS name 3-(azepan-1ylsulfonyl)-N-(3-nitrophenyl) benzamide were purchased from Sigma-Aldrich and Cayman Chemicals. Rapamycin and Staurosporine were from Santa Cruz Biotechnology (Santa Cruz, CA).

\section{Antibodies}

$\beta$-Actin (8H10D10) and $\alpha$-tubulin (DM1A) mouse $\mathrm{mAb}$, Merlin (D1D8), acetylated-lysine (A-K $\left.{ }^{2}-100\right)$, Acetyl- $\alpha$-tubulin (Lys40) (D20G3)XP, SIRT1(D1D7), SIRT3(D22A3), SIRT5(D8C3), SIRT7(D3K5A) and $\mathrm{LC} 3 \mathrm{~B}(\mathrm{D} 11) \mathrm{XP}$ rabbit $\mathrm{mAb}, \mathrm{HMGB} 1$ rabbit $\mathrm{Ab}$ were purchased from Cell Signaling (Danvers, MA). GAPDH $\mathrm{mAb}$ was from Millipore. SIRT2 rabbit $\mathrm{Ab}$ was from Sigma-Aldrich. S-100 rabbit Ab was purchased from Dako
Cytomation (Glostrup, Denmark). Secondary antibodies, peroxidase-conjugated goat anti-mouse IgG and goat antirabbit-IgG, were purchased from Pierce, Thermo Fisher (Rockford, IL). Goat anti-rabbit-IgG Alexa Fluor488-and -Fluor546 conjugated antibodies were purchased from Invitrogen (Grand Island, NY).

\section{Mouse Schwann Cell Cultures}

MSC were cultured on $200 \mu \mathrm{g} / \mathrm{ml}$ poly-L-lysine hydrobromide (PLL, Sigma-Aldrich), and $10 \mu \mathrm{g} / \mathrm{ml}$ Laminin (Invitrogen) coated 60 or $100-\mathrm{mm}$ Corning dishes. MSC growth medium: DMEM:F12 1:1 (Gibco) plus 1X-N2 supplement (Gibco), $2 \mu \mathrm{M}$ forskolin, $10 \mathrm{ng}$ / $\mathrm{ml}$ neuregulin and 1\% Penicillin/Streptomycin (Gibco).

\section{Merlin-Mutant Mouse Schwann Cell Culture}

Merlin-mutant MSC generated in the lab were cultured in CellBIND-Corning $100 \mathrm{~mm}$ dishes. Merlinmutant MSC growth medium was DMEM/F12 1:1 (Gibco); 1X-N2 supplement (Invitrogen) and 1\% Penicillin/Streptomycin. All protocols are in accordance with guidelines of and approved by the University of Central Florida (UCF) Institutional Animal Care and Use Committee (IACUC). UCF vivarium is Internationalcertified by the Association for Assessment and Accreditation of Laboratory Animal Care.

\section{Human Schwann Cell and HEI193 Cell Cultures}

Vials of frozen human SCs were thawed and seeded on coated $60 \mathrm{~mm}$ Corning plates $(200 \mu \mathrm{g} / \mathrm{ml}$ PLL and $50 \mu \mathrm{g} / \mathrm{ml}$ Laminin) containing D10M growth medium: DMEM (Gibco) plus 10\% heat inactivated fetal bovine serum (HIFBS, HyClone, Logan, UT), $2 \mu \mathrm{M}$ Forskolin (Sigma), $0.02 \mathrm{mg} / \mathrm{ml}$ Pituitary Extract (Biomedical Tech. Inc) and 1\% Penicillin/Streptomycin (Gibco). HEI193 cells were purchased from ATCC (Manassas, VA). HEI193 growth medium: DMEM plus 10\% HIFBS, 1\% Penicillin/ Streptomycin.

\section{Western Blot Analysis}

SCs were lysed in modified RIPA buffer $(25 \mathrm{mM}$ Tris- $\mathrm{HCl} \mathrm{pH} 7.6 ; 150 \mathrm{mM} \mathrm{NaCl}$ : 1\% Triton X-100, 1\% Sodium dodecyl sulfate (SDS) with protease inhibitor cocktail and phosphatase inhibitor cocktails 2 and 3, Sigma-Aldrich). To remove cell debris, lysates were centrifuged at $15,000 \mathrm{rpm}$ for $10 \mathrm{~min}$ at $4^{\circ} \mathrm{C}$. Protein concentration of the supernatant was determined with the DC Assay (BioRad, Hercules, CA). 10-15 $\mu \mathrm{g}$ of sample protein was resolved in 4-20\% polyacrylamide gels (Pierce), transferred to a polyvinylidene fluoride (PVDF) 
membrane (Immobilon-P, Millipore, Bedford, MA), blocked with $5 \%$ BSA and incubated overnight at $4{ }^{\circ} \mathrm{C}$ with anti- acetylated-lysine (1:500), Acetyl- $\alpha$-tubulin (Lys40) $(1: 1,000), \alpha$-tubulin $(1: 1,500), \quad$ SIRT1(1:500), SIRT2 (1:500), SIRT3(1:500), SIRT5(1:500), SIRT7(1:500), HMGB1 (1:500) for cell lysates and at (1:250) for concentrated medium, GAPDH (1:10,000), LC3B (1:500) and $\beta$-Actin $(1: 15,000)$ primary antibodies, followed by their corresponding secondary antibodies at 1:20,000. Quantification of western blots was done by densitometry using NIH ImageJ software.

\section{Immunofluorescence}

Control and merlin-mutant MSC were plated on coated German glass coverslips $(200 \mu \mathrm{g} / \mathrm{ml}$ PLL and $10 \mu \mathrm{g} / \mathrm{ml}$ Laminin) and immunostained as previously described [24]. Images were acquired using a Zeiss LSM710 Confocal microscope with 3 spectral detection channels, 5 laser lines - 458, 488, 514,543 \& 633 nm, FL filter set 49 DAPI, EX G365, FL filter set 43 CY 3, FL filter set 38 Endow GFP, all shift free, EC Plan-Neofluar 40x/1.3 DIC WD=0.21 M27 objective lens, with the ZEN2009 software. Fluorescence signals were acquired on separate channels with identical parameters for each labeled protein from a single plane. Fluorescence intensity of the green channel was done using Volocity software. Pictures were processed with the ZEN2011 software in the same manner.

\section{Cell Viability Assay}

Viability of dose-response assays was assessed with the CellTiter-Fluor cell viability assay (Promega) following manufacturer's specifications. Normal MSC and merlin-mutant MSC were seeded at 5,000 cells/well in $20 \mu \mathrm{l}$ of phenol-red free growth medium in 384-well plates (black with clear bottom, CellBIND-Corning) and centrifuged $1 \mathrm{~min}$ at $500 \mathrm{rpm}$. Cultures were incubated at $37^{\circ} \mathrm{C}, 7 \% \mathrm{CO} 2$ until attachment (2.5-3.5 hours), then 5 $\mu 1$ of compound/vehicle solution was added to each well. Plates were quick spun again and returned to the incubator for 24 hours. Fluorescence was read with a Synergy H1 Hybrid plate reader (BioTek, Winooski, VT).

\section{Proliferation Assays}

For the 72 hours cell proliferation study, cell numbers were assessed with the Crystal Violet Assay as previously described [46]. Equal number of merlin-mutant MSC were seeded in 24-well plates and cell number was evaluated at $0 ; 24 ; 48$ and 72 hour time points. Absorbance at $595 \mathrm{~nm}$ was measured with a $\mu$ Quant plate reader (BioTek).
Rate of DNA synthesis was evaluated at 24 hours with the Click-iT EdU Microplate Assay (Invitrogen/ Life Technologies) as previously described [23]. Oregon green-488 and DAPI fluorescence was measured with a Synergy H1 hybrid plate reader.

\section{Cell Cycle Analysis}

The cell cycle was analyzed by flow cytometry with propidium iodine (PI) staining and BrdU/7AAD. PI staining was performed on ethanol fixed cells using PI/ RNase solution from Beckton-Dickinson and studied on a BD Canto-II flow cytometer. Histograms were analyzed with ModFit LT software (Verity Software House, Topsham, ME). For each sample, 10,000 events were collected and the diploid population was distributed across $\mathrm{G}_{0} / \mathrm{G}_{1}, \mathrm{~S}$ and $\mathrm{G}_{2} / \mathrm{M}$ phases.

The BrdU/7AAD assay kit was purchased from Beckton-Dickinson. Cells were seeded in 6-well plates and treated overnight with inhibitor/vehicle. On the next day, $10 \mu \mathrm{M}$ BrdU was added to the cultures for 3 hours, cells were harvested, fixed and analyzed following manufacturer's instruction. The BD Canto-II flow cytometer with the BD FACSDiva ${ }^{\mathrm{TM}}$ 6.1.3 software was use for acquisition and Kaluza 1.2 (Beckman Coulter) software for data analysis.

\section{Apoptosis Assays}

For caspases dependent apoptosis the caspase activity was assessed by the Apo-ONE homogeneous Caspase-3/7 assay (Promega) following manufacturer's specifications. For caspases dependent and/or independent apoptosis the membrane asymmetry was measured with the Violet Ratiometric Membrane Asymmetry Probe/Dead Cell Apoptosis Kit (Invitrogen/Life Technologies). Both assay were previously described [23].

\section{Cytotoxicity Assays}

Cytotoxicity was assayed in a 384 well plate format with 5,000 cells/well after 24 hours incubation with the inhibitors/vehicle with the CellTox Green assay (Promega), following the express, no-step addition at seeding method described by the manufacturer. Cells treated with $27 \mu \mathrm{g} / \mathrm{ml}$ digitonin were considered 100\% cytotoxicity control. Fluorescence $(\mathrm{Ex}=510 \mathrm{~nm} / \mathrm{Em}=532$ $\mathrm{nm}$ ) was measured on a Synergy H1 Hybrid plate reader.

\section{LDH Release Assay}

LDH released into the culture medium was measured with the CytoTox-ONE assay (Promega). Merllin-null MSC were seeded at 4,000 cell/well in 
384 well plates and were treated on the next day with increasing doses of inhibitor/vehicle and incubated for 4 hours. Positive $100 \%$ cytotoxicity control was $27 \mu \mathrm{g} /$ $\mathrm{ml}$ digitonin. Plate and assay reagent were equilibrated to room temperature for $25 \mathrm{~min}$ and after addition of $25 \mu \mathrm{l}$ of assay reagent, the plate is incubated at room temperature in the Synergy H1 Hybrid plate reader. No stop solution was used. Fluorescence $(\mathrm{Ex}=560 \mathrm{~nm} / \mathrm{Em}=590 \mathrm{~nm})$ was measured $5 \mathrm{~min}$ after the first column received reagent.

\section{HMGB1 Release Assessment}

Equal number of merlin-null MSC per well in CellBIND 6-well plates were cultured overnight. On the next day the medium was replaced with $2 \mathrm{ml}$ treatment medium containing SIRT2 inhibitors/vehicle in duplicate wells and incubated for 6 hours at $37^{\circ} \mathrm{C}, 7 \% \mathrm{CO} 2$. Then working on ice, media from duplicates were pooled and transferred to cold $15 \mathrm{ml}$ conical tubes, centrifuged for $5 \mathrm{~min}$ at $800 \mathrm{~g}, 4^{\circ} \mathrm{C}$. The supernatant $(\sim 4 \mathrm{ml})$ was syringe filtered with $0.45 \mu \mathrm{m}$ filter (Fisher), transferred to cold $7 \mathrm{ml}$ 9K MWCO Pierce concentrators (Thermo Scientific) and centrifuged for $30 \mathrm{~min}$ at $4,000 \mathrm{~g}, 4^{\circ} \mathrm{C}$. From 120 to 150 $\mu l$ of concentrated proteins were obtained and prepared for SDS-PAGE. When media was removed from cultures, the plates were washed once with ice cold PBS and cell were lysed with the modified RIPA buffer mentioned above; protein concentration was measured with the DC assay (BioRad). Equal volume of concentrated proteins from medium and $10 \mu \mathrm{g}$ of protein of cell lysates were analyzed by western blotting for HMGB1.

\section{Statistical Analysis}

GraphPad Prism version 5.0 for Windows (GraphPad, La Jolla, CA, USA) was used for statistical analysis and graph generation. AGK-2 and AK1 doseresponse experiments were analyzed by non-linear regression (four parameters). Experimental data from three independent experiments were statistically analyzed by one or two-way ANOVA with post-tests, as indicated for each experiment.

\section{ACKNOWLEDGMENTS}

We thank Dr. Marco Giovaninni for the $N f 2^{\text {flox2 }}$ ${ }_{f l o x 2}$ mice, Dr. Patrick Wood, The Miami Project to Cure Paralysis, Department of Neurological Surgery, University of Miami Miller School of Medicine (Miami, Florida, USA) for vials of cultured human Schwann cells, Tiffany Fabianic and Stephani Klingeman Plati for technical assistance, and Layton Smith and Siohban Malany of Sanford Burnham Medical Research Institute, Florida for pilot LOPAC screen. This work was supported in part by a DHHS/NIH (5R01DC10189) and a CTF-Drug Discovery
Award to CFV. AP is recipient of a Young Investigator Award from the Children's Tumor Foundation.

\section{CONFLICT OF INTEREST}

The authors reported no potential conflicts of interest.

\section{REFERENCES}

1. Evans DG. Neurofibromatosis type 2 (NF2): a clinical and molecular review. Orphanet journal of rare diseases. 2009; 4:16.

2. Asthagiri AR, Parry DM, Butman JA, Kim HJ, Tsilou ET, Zhuang Z and Lonser RR. Neurofibromatosis type 2. Lancet. 2009; 373(9679):1974-1986.

3. Fong B, Barkhoudarian G, Pezeshkian P, Parsa AT, Gopen $\mathrm{Q}$ and Yang I. The molecular biology and novel treatments of vestibular schwannomas. Journal of neurosurgery. 2011; 115(5):906-914.

4. Kalamarides M, Acosta MT, Babovic-Vuksanovic D, Carpen O, Cichowski K, Evans DG, Giancotti F, Hanemann CO, Ingram D, Lloyd AC, Mayes DA, Messiaen L, Morrison H, North K, Packer R, Pan D, et al. Neurofibromatosis 2011: a report of the Children's Tumor Foundation annual meeting. Acta neuropathologica. 2012; 123(3):369-380.

5. Li W, Cooper J, Karajannis MA and Giancotti FG. Merlin: a tumour suppressor with functions at the cell cortex and in the nucleus. EMBO reports. 2012; 13(3):204-215.

6. Stamenkovic I and Yu Q. Merlin, a "magic" linker between extracellular cues and intracellular signaling pathways that regulate cell motility, proliferation, and survival. Current protein \& peptide science. 2010; 11(6):471-484.

7. North BJ and Verdin E. Sirtuins: Sir2-related NADdependent protein deacetylases. Genome biology. 2004; 5(5):224.

8. Hawse WF, Hoff KG, Fatkins DG, Daines A, Zubkova OV, Schramm VL, Zheng W and Wolberger C. Structural insights into intermediate steps in the Sir2 deacetylation reaction. Structure. 2008; 16(9):1368-1377.

9. Beirowski B, Gustin J, Armour SM, Yamamoto H, Viader A, North BJ, Michan S, Baloh RH, Golden JP, Schmidt RE, Sinclair DA, Auwerx J and Milbrandt J. Sir-two-homolog 2 (Sirt2) modulates peripheral myelination through polarity protein Par-3/atypical protein kinase C (aPKC) signaling. Proceedings of the National Academy of Sciences of the United States of America. 2011; 108(43):E952-961.

10. North BJ, Marshall BL, Borra MT, Denu JM and Verdin E. The human Sir2 ortholog, SIRT2, is an NAD+-dependent tubulin deacetylase. Mol Cell. 2003; 11(2):437-444.

11. Vaquero A, Scher MB, Lee DH, Sutton A, Cheng HL, Alt FW, Serrano L, Sternglanz R and Reinberg D. SirT2 is a histone deacetylase with preference for histone H4 Lys 16 
during mitosis. Genes \& development. 2006; 20(10):12561261.

12. Yang MH, Laurent G, Bause AS, Spang R, German N, Haigis MC and Haigis KM. HDAC6 and SIRT2 regulate the acetylation state and oncogenic activity of mutant K-RAS. Molecular cancer research : MCR. 2013; doi:10.1158/15417786.MRC-13-0040-T.

13. Liu L, Arun A, Ellis L, Peritore C and Donmez G. Sirtuin 2 (SIRT2) enhances 1-methyl-4-phenyl-1,2,3,6tetrahydropyridine (MPTP)-induced nigrostriatal damage via deacetylating forkhead box O3a (Foxo3a) and activating Bim protein. The Journal of biological chemistry. 2012; 287(39):32307-32311.

14. Narayan N, Lee IH, Borenstein R, Sun J, Wong R, Tong G, Fergusson MM, Liu J, Rovira, II, Cheng HL, Wang G, Gucek M, Lombard D, Alt FW, Sack MN, Murphy E, et al. The NAD-dependent deacetylase SIRT2 is required for programmed necrosis. Nature. 2012; 492(7428):199-204.

15. Outeiro TF, Kontopoulos E, Altmann SM, Kufareva I, Strathearn KE, Amore AM, Volk CB, Maxwell MM, Rochet JC, McLean PJ, Young AB, Abagyan R, Feany MB, Hyman BT and Kazantsev AG. Sirtuin 2 inhibitors rescue alpha-synuclein-mediated toxicity in models of Parkinson's disease. Science. 2007; 317(5837):516-519.

16. Chopra V, Quinti L, Kim J, Vollor L, Narayanan KL, Edgerly C, Cipicchio PM, Lauver MA, Choi SH, Silverman RB, Ferrante RJ, Hersch S and Kazantsev AG. The sirtuin 2 inhibitor AK-7 is neuroprotective in Huntington's disease mouse models. Cell reports. 2012; 2(6):1492-1497.

17. He X, Nie H, Hong Y, Sheng C, Xia W and Ying W. SIRT2 activity is required for the survival of C6 glioma cells. Biochemical and biophysical research communications. 2012; 417(1):468-472.

18. Hiratsuka M, Inoue T, Toda T, Kimura N, Shirayoshi Y, Kamitani H, Watanabe T, Ohama E, Tahimic CG, Kurimasa A and Oshimura M. Proteomics-based identification of differentially expressed genes in human gliomas: downregulation of SIRT2 gene. Biochemical and biophysical research communications. 2003; 309(3):558-566.

19. Kim HS, Vassilopoulos A, Wang RH, Lahusen T, Xiao Z, Xu X, Li C, Veenstra TD, Li B, Yu H, Ji J, Wang XW, Park SH, Cha YI, Gius D and Deng CX. SIRT2 maintains genome integrity and suppresses tumorigenesis through regulating APC/C activity. Cancer cell. 2011; 20(4):487499.

20. Li Y, Nie H, Wu D, Zhang J, Wei $\mathrm{X}$ and Ying W. Poly(ADP-ribose) polymerase mediates both cell death and ATP decreases in SIRT2 inhibitor AGK2-treated microglial BV2 cells. Neuroscience letters. 2013; 544:36-40.

21. Zhang Y, Au Q, Zhang M, Barber JR, Ng SC and Zhang B. Identification of a small molecule SIRT2 inhibitor with selective tumor cytotoxicity. Biochemical and biophysical research communications. 2009; 386(4):729-733.

22. Giovannini M, Robanus-Maandag E, van der Valk M,
Niwa-Kawakita M, Abramowski V, Goutebroze L, Woodruff JM, Berns A and Thomas G. Conditional biallelic Nf2 mutation in the mouse promotes manifestations of human neurofibromatosis type 2 . Genes \& development. 2000; 14(13):1617-1630.

23. Petrilli A, Copik A, Posadas M, Chang LS, Welling DB, Giovannini $\mathrm{M}$ and Fernandez-Valle C. LIM domain kinases as potential therapeutic targets for neurofibromatosis type 2 . Oncogene. 2013; doi:10.1038/onc.2013.320.

24. Thaxton C, Bott M, Walker B, Sparrow NA, Lambert S and Fernandez-Valle C. Schwannomin/merlin promotes Schwann cell elongation and influences myelin segment length. Molecular and cellular neurosciences. 2011; 47(1):1-9.

25. Hung G, Li X, Faudoa R, Xeu Z, Kluwe L, Rhim JS, Slattery $\mathrm{W}$ and Lim D. Establishment and characterization of a schwannoma cell line from a patient with neurofibromatosis 2. International journal of oncology. 2002; 20(3):475-482.

26. Kroemer G, Galluzzi L, Vandenabeele P, Abrams J, Alnemri ES, Baehrecke EH, Blagosklonny MV, ElDeiry WS, Golstein P, Green DR, Hengartner M, Knight RA, Kumar S, Lipton SA, Malorni W, Nunez G, et al. Classification of cell death: recommendations of the Nomenclature Committee on Cell Death 2009. Cell death and differentiation. 2009; 16(1):3-11.

27. Klionsky DJ, Abeliovich H, Agostinis P, Agrawal DK, Aliev G, Askew DS, Baba M, Baehrecke EH, Bahr BA, Ballabio A, Bamber BA, Bassham DC, Bergamini E, Bi X, Biard-Piechaczyk M, Blum JS, et al. Guidelines for the use and interpretation of assays for monitoring autophagy in higher eukaryotes. Autophagy. 2008; 4(2):151-175.

28. Dryden SC, Nahhas FA, Nowak JE, Goustin AS and Tainsky MA. Role for human SIRT2 NAD-dependent deacetylase activity in control of mitotic exit in the cell cycle. Mol Cell Biol. 2003; 23(9):3173-3185.

29. Inoue T, Nakayama Y, Yamada H, Li YC, Yamaguchi S, Osaki M, Kurimasa A, Hiratsuka M, Katoh M and Oshimura M. SIRT2 downregulation confers resistance to microtubule inhibitors by prolonging chronic mitotic arrest. Cell Cycle. 2009; 8(8):1279-1291.

30. North BJ and Verdin E. Interphase nucleo-cytoplasmic shuttling and localization of SIRT2 during mitosis. PLoS One. 2007; 2(8):e784.

31. Pandithage R, Lilischkis R, Harting K, Wolf A, Jedamzik B, Luscher-Firzlaff J, Vervoorts J, Lasonder E, Kremmer E, Knoll B and Luscher B. The regulation of SIRT2 function by cyclin-dependent kinases affects cell motility. J Cell Biol. 2008; 180(5):915-929.

32. Nie H, Chen H, Han J, Hong Y, Ma Y, Xia W and Ying W. Silencing of SIRT2 induces cell death and a decrease in the intracellular ATP level of PC12 cells. International journal of physiology, pathophysiology and pharmacology. 2011; 3(1):65-70.

33. Yuan Q, Yang H, Cheng C, Li C, Wu X, Huan W, Sun 
H, Zhou Z, Wang Y, Zhao Y, Lu X and Shen A. beta1,4-Galactosyltransferase I involved in Schwann cells proliferation and apoptosis induced by tumor necrosis factor-alpha via the activation of MAP kinases signal pathways. Molecular and cellular biochemistry. 2012; 365(1-2):149-158.

34. Tao T, Ji Y, Cheng C, Yang H, Liu H, Sun L, Qin Y, Yang J, Wang $\mathrm{H}$ and Shen A. Tumor necrosis factor-alpha inhibits Schwann cell proliferation by up-regulating Srcsuppressed protein kinase C substrate expression. Journal of neurochemistry. 2009; 111(3):647-655.

35. Tang X, Wang Y, Zhou S, Qian T and Gu X. Signaling pathways regulating dose-dependent dual effects of TNFalpha on primary cultured Schwann cells. Molecular and cellular biochemistry. 2013; 378(1-2):237-246.

36. Dan L, Klimenkova O, Klimiankou M, Klusman JH, van den Heuvel-Eibrink MM, Reinhardt D, Welte K and Skokowa J. The role of sirtuin 2 activation by nicotinamide phosphoribosyltransferase in the aberrant proliferation and survival of myeloid leukemia cells. Haematologica. 2012; 97(4):551-559.

37. Maxwell MM, Tomkinson EM, Nobles J, Wizeman JW, Amore AM, Quinti L, Chopra V, Hersch SM and Kazantsev AG. The Sirtuin 2 microtubule deacetylase is an abundant neuronal protein that accumulates in the aging CNS. Human molecular genetics. 2011; 20(20):3986-3996.

38. Harting $\mathrm{K}$ and Knoll B. SIRT2-mediated protein deacetylation: An emerging key regulator in brain physiology and pathology. European journal of cell biology. 2010; 89(2-3):262-269.

39. Heltweg B, Gatbonton T, Schuler AD, Posakony J, Li H, Goehle S, Kollipara R, Depinho RA, Gu Y, Simon JA and Bedalov A. Antitumor activity of a small-molecule inhibitor of human silent information regulator 2 enzymes. Cancer research. 2006; 66(8):4368-4377.

40. Hubbert C, Guardiola A, Shao R, Kawaguchi Y, Ito A, Nixon A, Yoshida M, Wang XF and Yao TP. HDAC6 is a microtubule-associated deacetylase. Nature. 2002; 417(6887):455-458.

41. Zuo Q, Wu W, Li X, Zhao L and Chen W. HDAC6 and SIRT2 promote bladder cancer cell migration and invasion by targeting cortactin. Oncology reports. 2012; 27(3):819824.

42. Burns SS, Akhmametyeva EM, Oblinger JL, Bush ML, Huang J, Senner V, Chen CS, Jacob A, Welling DB and Chang LS. Histone deacetylase inhibitor AR-42 differentially affects cell cycle transit in meningeal and meningioma cells, potently inhibiting NF2-deficient meningioma growth. Cancer Res.2013;73(2):792-803.

43. Bush ML, Oblinger J, Brendel V, Santarelli G, Huang J, Akhmametyeva EM, Burns SS, Wheeler J, Davis J, Yates CW, Chaudhury AR, Kulp S, Chen CS et al. AR42, a novel histone deacetylase inhibitor, as a potential therapy for vestibular schwannomas and meningiomas. Neuro Oncol. 2011;13(9):983-99.
44. Jacob A, Oblinger J, Bush ML, Brendel V, Santarelli G, Chaudhury AR, Kulp S, La Perle KM, Chen CS, Chang LS and Welling DB. Preclinical validation of AR42, a novel histone deacetylase inhibitor, as treatment for vestibular schwannomas. The Laryngoscope. 2012; 122(1):174-189.

45. Chen CS, Weng SC, Tseng PH, Lin HP and Chen CS. Histone acetylation-independent effect of histone deacetylase inhibitors on Akt through the reshuffling of protein phosphatase 1 complexes. The Journal of biological chemistry. 2005; 280(46):38879-38887.

46. Iacovelli J, Lopera J, Bott M, Baldwin E, Khaled A, Uddin $\mathrm{N}$ and Fernandez-Valle C. Serum and forskolin cooperate to promote G1 progression in Schwann cells by differentially regulating cyclin D1, cyclin E1, and p27Kip expression. Glia. 2007; 55(16):1638-1647. 